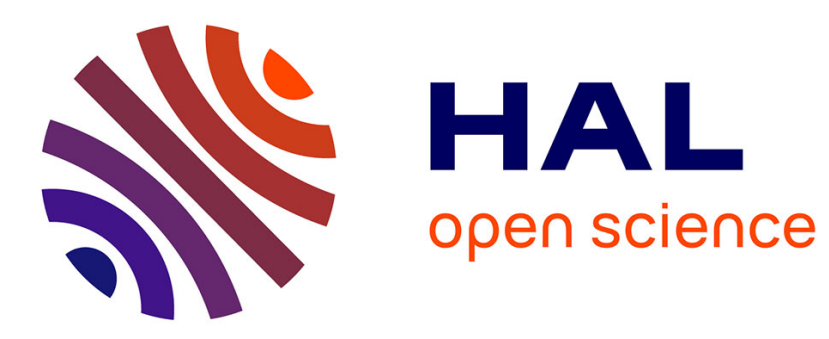

\title{
Estimate of hepatocellular carcinoma incidence in patients with alcoholic cirrhosis
}

Nathalie Ganne-Carrié, Cendrine Chaffaut, Valérie Bourcier, Isabelle Archambeaud, Jean-Marc Perarnau, Fréderic Oberti, Dominique Roulot, Christophe Moreno, Alexandre Louvet, Thông Dao, et al.

\section{To cite this version:}

Nathalie Ganne-Carrié, Cendrine Chaffaut, Valérie Bourcier, Isabelle Archambeaud, Jean-Marc Perarnau, et al.. Estimate of hepatocellular carcinoma incidence in patients with alcoholic cirrhosis. Journal of Hepatology, 2018, 69 (6), pp.1274-1283. 10.1016/j.jhep.2018.07.022 . hal-01880156

\section{HAL Id: hal-01880156 \\ https://hal-univ-rennes1.archives-ouvertes.fr/hal-01880156}

Submitted on 9 Nov 2018

HAL is a multi-disciplinary open access archive for the deposit and dissemination of scientific research documents, whether they are published or not. The documents may come from teaching and research institutions in France or abroad, or from public or private research centers.
L'archive ouverte pluridisciplinaire HAL, est destinée au dépôt et à la diffusion de documents scientifiques de niveau recherche, publiés ou non, émanant des établissements d'enseignement et de recherche français ou étrangers, des laboratoires publics ou privés. 


\section{Estimate of hepatocellular carcinoma's incidence in patients with alcoholic cirrhosis}

Short title: HCC incidence in alcoholic cirrhosis

Nathalie Ganne-Carrié ${ }^{1,2}{ }_{6}{ }^{3}$, Cendrine Chaffaut ${ }^{4}$, Valérie Bourcier ${ }^{1}$, Isabelle Archambeaud, Jean-Marc Perarnau , Frédéric Oberti ${ }^{7}$, Dominique Roulot ${ }_{12}^{2,8},{ }_{14}$ Christophe Moreno, Alexandre Louvet ${ }_{16}^{10}$, Thông Dao ${ }_{17}^{11}$, Romain Moirand $^{12}$, Odile Goria ${ }^{13}$, Eric Nguyen-Khac ${ }^{14}$, Nicolas Carbonell ${ }^{15}$, Térésa Antonini ${ }^{16}$, Stanislas Pol ${ }^{17}$, Victor de Ledinghen ${ }^{18}$, Violaine Ozenne ${ }^{19}$, Jean Henrion ${ }^{20}$, Jean-Marie Péron ${ }^{21}$, Albert Tran ${ }^{22}$, Gabriel Perlemuter $^{23}$, Xavier Amiot ${ }^{24}$, Jean-Pierre Zarski ${ }^{25}$, Michel Beaugrand ${ }^{1}$, Sylvie Chevret for CIRRAL Group.

${ }^{1}$ AP-HP, Hôpital Jean Verdier, Liver Unit, Bondy, France; ${ }^{2}$ University Paris 13, Sorbonne Paris Cité, "équipe labellisée Ligue Contre le Cancer" F-93000 Bobigny; ${ }_{4}^{3}$ Inserm, UMR-1162 «Functional Genomics of solid tumors », F-75010, Paris, France. S SBIM, APHP, Hôpital Saint-Louis, Paris, Inserm, UMR-1153, ECSTRA Team, Paris, France; Liver Unit, ${ }_{6}^{4}$ CHU, Nantes, France; Liver Unit, University Hospital, Tours, France; ${ }_{9}^{7}$ Liver Unit, University Hospital, Angers, France; ${ }^{8}$ Liver Unit, APHP, Avicenne, Bobigny;France; ${ }_{11}^{9}$ Liver unit, CUB Hôpital Erasme, Université Libre de Bruxelles, Belgium, ${ }^{10}$ Liver Unit, University Hospital, Lille, France, ${ }^{11}$ Liver Unit, University Hospital, Caen, France: ${ }^{12}$ Liver Unit, University Hospital, Rennes, France ${ }^{13}$ Liver Unit, University Hospital, Rouen, France ${ }^{14}$ Liver Unit, University Hospital, Amiens, France, ${ }^{15}$ Liver Unit, APHP, CHU Saint-Antoine, Paris, France, 16 Liver Unit, APHP, CHU Paul Brousse, Villejuif, France; 17 Université Paris Descartes; APHP, Liver Unit, Hôpital Cochin; INSERM U1223, Institut Pasteur, Paris, France; ${ }_{20}^{18}$ Hepatology Unit, University Hospital, CHU Bordeaux, France; ${ }_{21}^{19}$ Liver Unit, APHP, CHU Lariboisière, Paris, France; ${ }^{20}$ Liver Unit, University Hospital, Haine Saint-Paul, Belgium, ${ }^{21}$ Liver Unit, Universitary Hospital Purpan, University Paul Sabatier III, Toulouse ; ${ }^{22}$ Institut National de la Santé et de la Recherche Médicale (INSERM), U1065, Team 8, "Hepatic Complications in Obesity", Nice, F-06204, Cedex 3, France, University Hospital of Nice, Digestive Centre, Nice, F-06202, Cedex 3, France ; ${ }_{23}^{23}$ Liver Unit, University Hospital, Béclère, APHP, Clamart, France; ${ }^{24}$ Liver Unit, APHP, CHU Tenon, Paris, France; Clinique d'hépato-gastroentérologie pôle Digidune CHU de Grenoble, France

Declaration of interests: Prof. Ganne-Carrié received honoraria from Bayer, Bristol-Myers Squibb, and Gilead. Prof. Zarski consults for and is on the speakers' bureaus of Gilead, Bristol-Myers Squibb, Janssen, Siemens and MSD. He consults for AbbVie. Prof. Pol was a speaker for GSK, BMS, Boehringer Ingelheim, Janssen Gilead, Roche, MSD, Sanofi, Novartis, Vertex and Abbvie. He received grants from Bristol-Myers Squibb, Gilead, Roche, and MSD. He consults for Gilead, BristolMyers Squibb, Roche, Boehringer Ingelheim, Vertex, Janssen, AbbVie, Sanofi, Novartis and GlaxoSmithKline.

Financial support: The promoter of the study was the Assistance Publique des Hôpitaux de Paris (APHP), and the cohort was funded by the Institut National du Cancer (INCa).

\section{Author's Contributions:}

Study concept and design: Nathalie Ganne-Carrié, Sylvie Chevret.

Acquisition of data: Valérie Bourcier, Nathalie Ganne-Carrié, Isabelle Archambaud, Jean-Marc Perarnau, Frédéric Oberti, Dominique Roulot, Christophe Moreno, Alexandre Louvet, Thông Dao, Romain Moirand, Odile Goria, Eric Nguyen-Khac, Nicolas Carbonell, Teresa Antonini, Stanislas Pol, Victor de Ledinghen, Violaine Ozenne, Jean Henrion, Jean-Marie Péron, Albert Tran, Gabriel Perlemuter Xavier Amiot, Jean-Pierre Zarski, Cendrine Chaffaut, Sylvie Chevret

Analysis and interpretation of data: Valérie Bourcier, Cendrine Chaffaut, Sylvie Chevret, Michel Beaugrand, Nathalie Ganne-Carrié

Drafting of the manuscript: Nathalie Ganne-Carrié, Cendrine Chaffaut, Sylvie Chevret. 
Critical revision of the manuscript for important intellectual content: Valérie Bourcier, Cendrine Chaffaut, Sylvie Chevret, Nathalie Ganne-Carrié, Michel Beaugrand.

Statistical analysis: Cendrine Chaffaut and Sylvie Chevret

Obtained funding: Nathalie Ganne-Carrié

Administrative, technical and material support: Valérie Bourcier, Cendrine Chaffaut, Sylvie Chevret Study supervision: Valérie Bourcier, Cendrine Chaffaut, Sylvie Chevret, Nathalie Ganne-Carrié

Keywords: alcoholic liver disease, compensated cirrhosis, HCC, competing risk analysis.

Electronic word count (including abstract, references, legends to figures, tables): 6680

Figures: 4, Tables: 3 , Supplementary tables: 3

Registration: The study was registered at ClinicalTrials.gov (number NCT01213927) and the full protocol is available at the following link: https://clinicaltrials.gov/ct2/show/NCT01213927

Role of the sponsor: The funding sponsor had no role in the design or execution of the study; in the collection, management, analysis, or interpretation of data; or in the preparation, review or approval of the manuscript.

Abbreviations: AFP: alfa-fetoprotein; BMI: Body Mass Index; EFS: event-free survival; HBV: hepatitis B virus; HCV: hepatitis C virus; HCC: hepatocellular carcinoma; HR: hazard ratio; IQR: interquartile range; OS: overall survival; US: abdominal ultrasonography examination.

\section{ABSTRACT (275 words/ 275)}

Background and aims: The CIRRAL cohort aimed to assess the burden of complications in patients with alcoholic cirrhosis, particularly the occurrence of hepatocellular carcinoma (HCC).

Patients with biopsy-proven compensated alcoholic cirrhosis were included then prospectively followed. The main end point was the incidence of HCC. Secondary outcomes were incidence of hepatic focal lesions, overall survival (OS), liver-related mortality and event-free survival (EFS).

Results: From October 2010 to April 2016, 652 patients were included in 22 French and Belgian centers. During follow-up (median 29 months), HCC was diagnosed in 43 patients. With the limitation derived from the uncertainty of consecutive patients' inclusion and from a 
sizable proportion of dropouts (153/652), the incidence of HCC was 2.9 per 100 patients-year, and 1- and 2-year cumulative incidences of $1.8 \%$ and 5.2\%, respectively. Although $\mathrm{HCC}$ fulfilled the Milan criteria in 33 cases (77\%), only 24 patients $(56 \%)$ underwent a curative treatment. An explorative prognostic analysis showed that age, male gender, baseline AFP, bilirubin and prothrombin were significantly associated with the risk of $\mathrm{HCC}$ occurrence. Among 73 deaths, 61 had recorded cause and 27 were directely attributable to liver disease. At 2 years, OS, EFS and cumulative incidences of liver-related deaths were 93\% (95\% CI: 90.5-95.4), 80.3\% (95\%CI: 76.9-83.9), and 3.2\% (95\%CI: 1.6-4.8) respectively.

Conclusion: This large prospective cohort incompletely representative of the whole population with alcoholic cirrhosis showed: a) an annual incidence of HCC up to 2.9 per 100 patients-year, suggesting that surveillance might be cost effective in these patients; b) a high proportion of HCC detected within the Milan criteria, but only one-half of detected HCC cases were referred for curative treatments; c) a 2-year mortality up to $7 \%$.

\section{Lay Summary}

Cirrhosis is a risk factor for primary liver cancer leading to recommandations for periodical screening. However, in case of an alcoholic origin of the liver disease, the rational of periodical screening for $\mathrm{HCC}$ is controversial, as registry and databased studies have suggested low incidence of HCC in these patients and a high competitive mortality. With the limitation of a possible selection and attrition bias, this large cohort of unambiguously biopsy proven alcoholic cirrhosis prospectively screened for HCC demonstrated a high annual incidence of HCC (2.9\%) and a high percentage of small cancers theorically eligible for curative treatment, suggesting that alcoholic origin of the disease shouldn't rule out patiens for screening.

\section{Introduction}


Primary liver cancer has a high incidence in Europe, especially in France, which has almost 9000 cases per year (1). In more than $90 \%$ of cases, hepatocellular carcinoma (HCC) occurs in patients with cirrhosis. Alcohol is the leading cause of the underlying cirrhosis that is associated with HCC in France; it is responsible for more than $60 \%$ of the cases (2), which is much higher than the proportion of cases from hepatitis $\mathrm{B}$ and $\mathrm{C}$ and non-alcoholic steatohepatitis. Although precise data are lacking, as France has lost its longstanding European leadership in alcohol consumption, one can speculate that alcoholic cirrhosis is currently the main cause of HCC occurrence in Europe, and it will gain an even more important predominance as alcoholic consumption is on the rise in many countries, while active viral infection is on the decline.

Despite recommendations (3-5), including those for alcoholic liver disease (6), screening for HCC in patients with cirrhosis is poorly performed in practice and is even contested, particularly in alcoholics (7). One matter of disagreement is the incidence of HCC in these patients. Based on medico-economic studies, the threshold of HCC incidence that is associated with cost-effectiveness is $1.5 \%$ per year, and surveillance should only be undertaken in patients whose risk of HCC is over this threshold. There are few and conflicting data regarding the incidence of $\mathrm{HCC}$ in patients with alcoholic cirrhosis. A retrospective Danish study, using its National Patient Registry (8), and the United Kingdom's General Practice Research Database population-based study (9) have demonstrated a low risk of HCC (between 0.25 and $0.50 \%$ per year in the Danish study and less than $2 \%$ at 10 years in the British study), raising some doubts regarding the opportunity of screening for HCC in these patients. In contrast, a small but very homogeneous, Spanish cohort of patients with alcoholic cirrhosis prospectively followed for HCC occurrence showed a high incidence of HCC (up to $2.6 \%$ per year) (10), which suggests that the implementation of a surveillance program for the early diagnosis of HCC is warranted in these patients. 
The French and Belgian CIRRAL prospective cohort was constituted to assess the natural history of alcohol-related compensated cirrhosis based on rigorous, multicenter, protocoldriven, systematic data collection and the analysis of predefined outcomes.

The main purpose of this first report was to assess the incidence of HCC in a wellcharacterized population of patients with biopsy-proven alcoholic cirrhosis who were periodically followed and screened for HCC using competing risks analysis. The secondary objectives were to develop a prediction model for HCC occurrence and to assess the course of the cirrhosis and extra-hepatic events.

\section{Patients and methods}

The promoter of this study was the Assistance Publique des Hôpitaux de Paris. The study was approved by an Ethics Committee (Comité de Protection des Personnes, Aulnay-sous-Bois, France) and registered at ClinicalTrials.gov (number NCT01213927). The trial protocol is available at the link https://clinicaltrials.gov/ct2/show/NCT01213927. Written informed consent was obtained from each patient before enrollment.

\section{Patient selection}

Patients were recruited from 28 French and Belgian clinical centers specialized in the management of chronic liver diseases (Figure 1a). Inclusion of patients started in October 2010 and was completed in April 2016 (predefined target enrollment). Consecutive patients fulfilling the six following criteria were selected: a) age older than 18 years; b) biopsy-proven cirrhosis, regardless of the time of biopsy; c) chronic alcohol abuse according to the World Health Organization criteria (more than 21 glasses per week for female and more than 28 glasses per week for male) that occurred for at least ten years and was considered the cause of chronic liver disease; d) no associated HBV (negative serum HBs Ag) or HCV infections (negative HCV serology) or other definite cause of cirrhosis; e) Child-Pugh class A disease at the time of enrollment; f) absence of previous primary liver cancer and baseline detectable 
suspected liver focal lesion on the pre-inclusion assessment.

\section{Study design}

Pre-inclusion assessment included the usual clinical and biological parameters; an abdominal ultrasonography (US) examination was also undertaken to rule out any baseline complications of cirrhosis, including primary liver cancer.

At inclusion, and then yearly for three years, serum biobanks (serum, plasma and DNA) were collected for further ancillary studies, and screening for ethanol consumption was detected by a breath test.

Patient information was recorded in an Electronic Case Report Form by a clinical research associate who was specifically dedicated to the CIRRAL cohort in each center. For all patients, past and ongoing alcohol or tobacco consumptions were quantified and recorded at inclusion. Past medical history and all events that occurred before enrollment were recorded at baseline.

On the basis of the International Diabetes Federation (11) updated in 2009, metabolic syndrome was diagnosed in case of the presence of at least 3 of the following features: abdominal obesity with increased waist circonference; serum lipids triglycerides $\geq 150 \mathrm{mg} / \mathrm{dL}$ $(1,7 \mathrm{mmol} / \mathrm{L})$, serum HDL-Cholesterol $<40 \mathrm{mg} / \mathrm{dL}(1,03 \mathrm{mmol} / \mathrm{L})$ in men or $<50 \mathrm{mg} / \mathrm{dL}(1,29$ $\mathrm{mmol} / \mathrm{L}$ ) in women, Blood pressure $\geq 130 \mathrm{~mm} \mathrm{Hg}$ systolic or $\geq 85 \mathrm{~mm} \mathrm{Hg}$ diastolic, Fasting serum glucose $\geq 100 \mathrm{mg} / \mathrm{dL}(5,6 \mathrm{mmol} / \mathrm{L})$.-

\section{Follow-up}

Patients were prospectively followed according to French guidelines (Haute Autorité de Santé), with an examination by Doppler US performed every 6 months by a radiologist with expertise in hepatic diseases in the hospital of the participating center or outside--A report was completed by each operator, mentioning the presence or absence of focal liver lesions. In addition, blood tests were performed at least yearly, and regular endoscopic surveillance was scheduled according to avalaible international and French guidelines: i) yearly in patients 
with decompensated cirrhosis or in those who had small varices; ii) at 2-year interval in patients with compensated cirrhosis who had no varices on screening endoscopy. In case of esophageal varices, preventive therapy was recommended either using beta-blockers or endoscopic ligation.

\section{Study Outcome Measures}

The main end point was the incidence of HCC from inclusion. Secondary outcome measures were the incidences of liver nodules, event-free survival (EFS), overall survival (OS) and liver-related mortality. EFS accounted for the first event (related either to liver or not) since enrollment, including death, regardless of cause.

All events that occurred during follow-up were recorded based on information obtained from the medical files of patients at each center but only the first event since enrolment was considered in the statistical analysis. Beside HCC, four predefined liver-related events were recorded as well as their severity, management, and outcome: 1. Ascites defined by clinical symptoms with increased abdominal volume and paracentesis requirement; 2. Jaundice defined by increased bilirubin serum level over $100 \mu \mathrm{mol} / \mathrm{L}$; 3. Encephalopathy defined according international criteria; 4. Gastro-intestinal bleeding defined by overt hemorrhage in relation with portal hypertension. In addition, all extra-hepatic events occurring during follow-up were recorded with the help of a drop-down menu with events proposal included in the eCRF for each visit (supplementary table 1). Bacterial infections were diagnosed as follows: rinary tract infection: more than 10 leucocytes per highpower field in urine and positive urine cultures or significant leucocyte count per field without positive cultures; Pneumonia : presence of radiologic evidence of consolidation, plus at least two of the following criteria: fever higher than $38^{\circ} \mathrm{C}$ or hypothermia less than $35^{\circ} \mathrm{C}$, dyspnea, cough and purulent sputum, pleuritic chest pain or signs of consolidation on physical examination; Spontaneous bacterial peritonitis : polymorphonuclear cell count in ascitic fluid >250/mm3;

Other systemic infection encompassed infections with systemic damage such as catheter- 
related infection (positive blood and catheter cultures) or osteoarticular infections (positive blood and/or bone biopsy or joint aspiration). Likely cause(s) of death were established. All treatments were recorded at inclusion, and any modifications during follow-up were noted. Patients were considered lost to follow-up in the absence of any news for 18 months. To reduce the number of patients lost to follow-up, a procedure was predefined. Phone calls and/ or recall letters were sent to patients and their general practionner in cases of 2 consecutive missed visits. In the absence of any evolutive information, the municipalities of birth were contacted in order to determine whether patients were deceased or not.

All recorded information during follow-up was secondarily monitored by a dedicated clinical research associate.

\section{HCC diagnosis and treatment}

In cases of focal liver lesion detected by US: i) echogenicity, number and diameter (classified as $<10 \mathrm{~mm}, 11-20 \mathrm{~mm}, 21-30 \mathrm{~mm}, 31-50 \mathrm{~mm}$, or $>50 \mathrm{~mm}$ ), as well as anatomic localization according to the Couinaud classification were reported; ii) portal vasculature (main trunk and branches), hepatic veins, and vena cava were systematically examined; iii) a diagnostic procedure using contrast-enhanced imaging (CT-scan or MRI), serum alpha-fetoprotein (AFP) assay or a guided biopsy was performed according to the 2005 AASLD guidelines (4), which were updated in 2011 (5).

HCC diagnosis was thus established either by histological examination performed by an experienced pathologist or by using probabilistic non-invasive criteria (mainly dynamic imaging showing early arterial hyper vascularization and portal washout) according to calendar time (before or after 2011). When the HCC diagnosis was established, treatment was decided through a multidisciplinary approach according to the AASLD (5) or EASL guidelines for HCC (3). Reports of imaging techniques showing liver focal lesions were secondarily reviewed by the two senior hepatologists from institution 1 (VB and NGC).

\section{Statistical analyses}


Analysis was performed at the reference date of January 11, 2017, to ensure independent administrative right censoring of the failure time data. Summary statistics, i.e., absolute and relative frequencies or median (interquartile range, IQR), were computed. The cumulative incidences of HCC and liver-related death were estimated in a competing risks framework, where deaths free of the event of interest were considered to be competing events. In order to explore the risk of false negative of liver ultrasound at inclusion, a sensitivity analysis excluding HCC that occurred within the first 6 months after inclusion was performed. EFS and OS were estimated by the Kaplan-Meier method; to assess the impact of violations in the underlying assumption of non-informative censoring due to potential study dropouts before the reference date, we imputed the event time of those subjects, and treat them as they have had an event occurred at the time of early withdrawal, resulting in a worst scenario, while considering that they were free of the event of interest at the end of scheduled follow-up resulting in a best scenario (12). Patients who underwent liver transplantation without HCC were censored for analysis at the date of transplantation given no data was recorded in the eCRF after such an event. Prognostic factors for HCC occurrence as selected from the literature (13) were evaluated by cause-specific Cox models as reported in Hsu et al (14). Univariate models were first fitted. To avoid overfitting, we included in a multivariable model, only the variables associated with the outcome at the $5 \%$ level unless low prevalence and variable selection used a backward stepwise procedure at the 5\% level. Beside baseline potential predictors, we also considered time-dependent covariates, namely current body mass index (BMI), as well as ongoing alcohol consumption and ongoing smoking. Missing values of covariates in the multivariable model were handled by multiple imputations with chained equations (15), based on $\mathrm{M}=30$ imputed complete datasets, with an estimated hazard ratio (HR) based on the average value of the regression coefficients. As a post-hoc sensitivity analysis, estimates were given after exluding patients with focal liver lesions at baseline or who developed HCC with the 6 first months. Point estimates are reported with 95\% 
confidence intervals (95\%CI). Analyses were performed on SAS 9.3 (SAS Inc., Cary, NC) and R3.0.2 (http://www.R-project.org/). This manuscript has been prepared according to the Transparent Reporting of multivariate prediction models for individual prognosis or diagnosis (TRIPOD) statement (16) (checklist included as supplementary table 2).

All co-authors had access to the study data and had reviewed and approved the final manuscript.

\section{Results}

\section{Inclusion period and data analysis}

From October 2010 to April 2016, 706 eligible patients who signed an informed consent were enrolled in 22 active centers out of 28 (Figure 1b). Among them, 54 were subsequently excluded from analysis after the revision of individual data due to either violation of the inclusion criteria $(n=48)$ or consent withdrawal $(n=6)$. Consequently, the further analyses address 652 patients (Figure 2).

\section{Baseline characteristics of patients (Table 1)}

Patients were mainly male (68\%), with a median age of 58.4 years [IQR: 51.2- 64.3]. At inclusion, median time since liver biopsy was 25.8 months [IQR: 8.6-70.8] and previous decompensation of their cirrhosis was observed in 416 (65\%) patients. Median BMI was 27.5 [IQR: 24.2-31.1] kg per square meter. Overweight or obesity (BMI > $25 \mathrm{~kg} /$ per square meter) and/or metabolic syndrome were recorded in 421 (67.5\%) and 114 (21\%) patients, respectively. Four hundred forty-one patients (67.6\%) were completely abstinent with a median duration of alcohol withdrawal of 24.7 months [IQR: 9.5-72] and 106 (16.9\%) still had a mild consumption $<7$ glasses / week. These declarations were in accordance with the absence of ethanol detection in the breath at enrollment in 410 (78\%) patients. Most patients were smokers (past history of tobacco, $n=221,35 \%$; active smokers, $n=258,41 \%$ ) and regular coffee consumers ( $\mathrm{n}=427,77 \%$; median: 2 cups/day; • 3 cups/day in 160/427). 
At enrolment, 44 patients $(6.7 \%)$ had a benign focal liver lesion, diagnosed either as "nonhepatocellular" in 23 patients (10 hemangiomas, 11 biliary cysts, 1 arteriovenous fistula, 1 steatosis focus) or as hepatocellular in 11 patients (3 dysplastic nodules, 8 regenerative nodules) and indeterminate, but not suspect for HCC, in 10 patients.

\section{Follow-up}

At the reference date of analysis in January 2017, the median follow-up was 29 months [IQR: 12.2- 41.4]. The overall compliance to surveillance was good, as assessed by a median time of 6.2 [IQR: 5.5-7.1] months between periodic abdominal ultrasonography. However, 153 patients $(23.5 \%)$ were lost to follow-up at the reference date, all free of events at the time of study dropout except one who developed HCC. Compared to regularly followed patients, those lost to follow-up had less severe liver disease with a lower rate of previous history of decompensation, less frequent endoscopic evidence of portal hypertension at enrollment and lower bilirubin serum level at baseline" (supplementary table 3).

\section{Liver focal lesions and primary liver cancer}

During the follow-up, focal liver lesions were observed in 125 patients, including 23 out of the 44 patients with focal liver lesions at baseline, and they were the first focal lesions in 102 additional patients (Figure 3). The 1-year and 2-year estimates of the cumulative incidence of focal liver lesions from enrollment were 13\% (95\%CI: 10.3- 15.7) and 20.8\% (95\%CI: 17.224.3), respectively (Figure 3a). Most of these focal liver lesions remained indeterminate or were considered benign. A diagnosis of HCC was established in 43 patients (6.6\%), with 10 cases in the first year, 15 within the second year, and 18 therafter. The incidence of HCC was 2.9 per 100 patients-year with estimates of cumulative incidence at 1 and 2 years of $1.8 \%$ (95\%CI: $0.7 ; 3)$ and 5.2\% (95\%CI: 3.2; 7.2), respectively (Figure 3a). Six patients with benign focal lesion at baseline developed an HCC, including 3 with biliary cyst, 1 with a dysplastic nodule, 1 regenerative nodule and 1 hyperechogenic nodule, all of them in another segment of the liver suggesting that HCC was not developed from the baseline focal lesion. 
After excluding those 46 patients with focal liver lesion at baseline $(n=44)$ or who developed an $\mathrm{HCC}$ within the 6 first months $(\mathrm{n}=2)$, the incidence of $\mathrm{HCC}$ was 2.6 per 100 patients-year with estimates of HCC cumulative incidence at 1 and 2 year of $1.2 \%$ (CI 95\%: 0.2-2.2) and $4.4 \%$ (CI95\%: 2.4- 6.3), respectively (Figure 3a). In sensitivity analyses, the estimated 1 year estimate of HCC cumulative incidence ranged from $1.4 \%$ (95\%CI: 0.7-2.7) in the best scenario where all dropouts were free of $\mathrm{HCC}$ at the end of scheduled follow-up, up to $7.3 \%$ (95\%CI: 5.1-9.3) in the worst scenario where all dropouts were actually HCC at the time of study dropout.

In patients with liver focal lesion, the cumulative incidence of HCC was higher in the first year following such a detection than thereafter, with $34 \mathrm{HCC}$ in the first year, 5 in the second year and 3 therafter (Figure $3 b$ ).

All HCC cases (except one) fulfilled the non-invasive criteria, and 8 HCCs were biopsyproven. Most HCCs were uni-nodular $(n=25,58 \%)$. The median diameter of the largest nodule was $23 \mathrm{~mm}$ [IQR: 16-29], and most $\mathrm{HCC}$ were $30 \mathrm{~mm}$ or less $(\mathrm{n}=33,77 \%)$. At diagnosis of HCC, 79.4\% of patients still had compensated cirrhosis (Child-Pugh A), while 20.6\% were classified B according to the Child-Pugh classification. Tumorous portal obstruction and a serum AFP level $>200 \mathrm{ng} / \mathrm{mL}$ were observed in 4 cases (9\%) and 2 cases (5\%), respectively. Overall, 33 (77\%) patients with HCC fulfilled the Milan criteria (Table 2). Only 24 patients received a first-line curative treatment (56\%) (transplantation $n=1$, resection $\mathrm{n}=5$, percutaneous ablation $\mathrm{n}=18$, including 1 patient who had a second-line liver transplantation 29 months after radiofrequency), while the others either had palliative treatments (embolization $n=8$, radiotherapy $n=1$, sorafenib $n=3$ ) or the best supportive care, including 6 patients with small $\mathrm{HCC}$ within the Milan criteria who were ruled out for curative treatment, either because of impaired performance status $(>2)$ or because of liver failure (Child-Pugh > B7 with ascites).

Secondary outcomes 
A total of 148 patients experienced at least one event related to liver disease. The first observed event was ascites, hepatic encephalopathy, gastrointestinal bleeding, or jaundice in 64 patients, $\mathrm{HCC}$ in 25 patients, bacterial infection in 21 patients, non-liver events related to alcohol in 10 patients and death in 28 patients, resulting in a 2-year cumulative incidence of liver decompensation of 12.4\% (95\%CI: 4.2-8.2) (Figure 4a). The 2 year EFS was estimated at $80.3 \%$ (95\%CI: 76.9-93.9).

Overall, 73 patients died during follow-up, with a 2-year survival of 93\% (95\%CI: 90.5-95.4) (Figure 4b); note that the estimated 2-year survival ranged from 82.2\% (95\%CI: 79-85.5) in the worst scenario of considering all study dropouts as deaths up to $93.7 \%$ (95\%CI: 91.7 95.8) in the best scenario assuming all dropouts as alive at 5 years, Among patients with known cause of death $(n=61 / 73)$, mortality was directly attributable to liver disease in 27 (44\%). Liver decompensation was the cause of death in 19 patients, HCC in 8 and extrahepatic disease in 34. Among the 43 patients with HCC, 14 died, with a 1-year survival rate of $64.3 \%$ (95\%CI: 48.3-85.5) after diagnosis (Figure 4c). Thus, among patients with known cause of death $(n=61 / 73)$, the 2-year cumulative incidence of liver-related death was $3.2 \%$ (95\%CI: 1.6-4.8); in contrast, the 2-year cumulative incidence of death from other causes was 2.5\% (95\% CI: 1.4-4.2) (Figure 4d).

\section{Predictive factors for HCC (Table 3).}

Univariate analysis selected 12 baseline variables as associated with the outcome, namely, age, gender, body mass index, diabetes, arterial hypertension, complete alcohol withdrawal, coffee consumption ( $>3$ cups daily), AFP, prothrombin time, bilirubin, albumin, and platelets.

Only 5 of these variables were selected as adding predictive information to each other by multivariable analysis. Four variables were associated with an increased cause-specific hazard of $\mathrm{HCC}$, namely, old age $(\mathrm{HR}=1.12,95 \% \mathrm{CI}, 1.07-1.18 ; \mathrm{p}<0.0001)$, male gender $(\mathrm{HR}=2.66$ (95\%CI, 1.12-6.32; $\mathrm{p}=0.027)$, high baseline AFP $(\mathrm{HR}=1.07,95 \% \mathrm{CI}, 1.02-1.12 ; \mathrm{p}=0.004)$ and bilirubin levels $(\mathrm{HR}=1.06,95 \% \mathrm{CI}, 1.102-1.096 ; \mathrm{p}=0.005)$, while high values of prothrombin 
time were associated with a decreased hazard of $\mathrm{HCC}(\mathrm{HR}=0.97,95 \% \mathrm{CI}, 0.94-0.99$; $\mathrm{p}=0.025)$. Stratifying the model on previous decompensation did not markedly affect those results (data not shown).

When incorporating time-dependent alcohol consumption, smoking and BMI, only ongoing consumption of alcohol was selected as predictive of $\mathrm{HCC}(\mathrm{HR}=1.98,95 \% \mathrm{CI}, 1.18-3.31)$ based on univariable models. However, after adjustment on the previously selected baseline predictors, neither ongoing alcohol consumption $(\mathrm{p}=0.36)$, nor active smoking $(0.88)$, nor current BMI $(\mathrm{p}=0.64)$ were predictive of the occurrence of HCC.

\section{Discussion}

The main result of the Franco-Belgian CIRRAL prospective cohort is the high incidence of HCC in patients with alcoholic cirrhosis (2.9 per 100 patient-year). This incidence rate is far beyond the threshold of $1.5 \%$ per year at which the cost-effectiveness of HCC screening has been considered disputable, thus suggesting that surveillance might be cost effective in these patients. The observed cumulative incidence at 1 year (1.8\%) may be underestimated due to the inclusion criteria and the criteria of diagnosis. Indeed, patients were included in the cohort based on the absence of any suspicious nodules, while the diagnosis of HCC was established for nodules larger than $1 \mathrm{~cm}$ in diameter. This diagnosis was mainly based on non-invasive criteria; diagnosis was made in cases of arterial hypervascularization that were associated with washout in the tardive venous phase, a pattern that is uncommon in very early HCC. Conversely, the 2-year cumulative incidence (5.2\%) is probably closer to reality. This incidence is in the same range as that observed in patients with HCV cirrhosis that were recruited in the French ANRS CO12 Cirvir cohort using similar inclusion criteria, that is, the absence of liver failure (Child-Pugh < B7) and the absence of suspicious nodules (17).

In this cohort of patients mainly with inactive cirrhosis and a controlled cause of liver disease (complete abstinence from alcohol or mild residual alcohol consumption in $84.5 \%$ of cases at 
enrollment), as previously described for virus-related cirrhosis after hepatitis $\mathrm{C}$ eradication $(18,19)$, the independent risk factors for HCC occurrence are host-related (age, sex) and linked to the baseline severity of cirrhosis (bilirubin, prothrombin time). In addition, the complete alcohol abstinence at enrollement was a protective factor against the further development of HCC in univariate analysis, suggesting that a firm recommendation of alcohol withdrawal is warranted in patients with alcoholic cirrhosis.

Conversely, in the small sub-group of patients with baseline active alcohol consumption, we didn’t observe a “dose-dependent risk for HCC occurrence.

Our study has numerous strengths that guarantee reliable results. First, patients were unambiguously identified with biopsy-proven cirrhosis and were selected with stringent criteria regarding liver function. In contrast to the Danish cohort (8), the absence of complications of cirrhosis at enrollment in the CIRRAL cohort limits competing risks, allowing us to analyze the long-term HCC risk. Second, the viral causes of chronic liver disease were ruled out. Third, the prospective design ensured an unbiased record of events during the follow-up of patients. Fourth, patients were closely screened for HCC by periodic abdominal ultrasonography with an overall good compliance. Finally, accurate statistical methods were accordingly based on competing risks analysis, and the multiple imputation technique was used for dealing with missing data (15).

As previously reported (20), a high incidence of focal liver lesions (9.7\% at 1 year and $17 \%$ at 2 years) was observed after short-term follow-up, indicating that approximately $10 \%$ of patients with alcoholic cirrhosis will need a recall policy to rule out malignancy every year.

However, a firm diagnosis of HCC was established in $25 \%$ of these patients. This incidence of focal liver lesions, already demonstrated to be greater than the incidence in patients with HCV-related cirrhosis (20), might be explained, at least in part, by the presence of steatosis, most of these nodules disappearing during follow-up. However, one cannot rule out that some might be dysplastic nodules or even small HCCs, due to the short follow-up. Interestingly, 
focal liver lesions observed at baseline disappeared in 19 out of 44 patients (43\%). The disappearance of liver nodules in cirrhotic is not unusual and was already reported by Trinchet et al (20). Interestingly, at the end of its randomized controlled trial comparing 3and 6-month periodicities for ultrasonographic surveillance of HCC in cirrhosis, and despite a long follow-up, about $45 \%$ of the detected nodules either disappeared or were considered to be of indeterminate nature, especially for those small up to $10 \mathrm{~mm}$ in diameter. Irregular steatosis sparing of some cirrhotic nodules can be seen by contrast imaging with hypoechoic and disappear later on.

One purpose of our study was to consider the potential benefit of screening in terms of access to a potentially curative treatment. Seventy-seven percent of the detected HCC were within the Milan criteria, but only $56 \%$ of patients with detected HCC (24/43) were treated with a curative intent. This result suggests that patients with alcoholic cirrhosis have reduced access to the curative treatment of HCC, as was previously emphasized in a French nationwide study showing that HCCs related to only alcohol were significantly less likely to receive curative treatments than HCCs related to only to $\mathrm{HCV}(20.6 \%$ vs $36.1 \%$, OR $0.45[0.42-0.50], \mathrm{p}<$ $0.001)$, regardless of the type of curative treatment (3.8\% vs $6.3 \%$ for transplantation, $8.1 \%$ vs $12.7 \%$ for surgery and $8.6 \%$ vs $17.1 \%$ for percutaneous ablation) (21). This finding could be due to the multiple extra-hepatic comorbidities that are common in this population, as previously described (22), and it was emphasized in our study by the rather high rate of extrahepatic death in a mostly compliant population. Nevertheless, even in patients who were contra-indicated for surgery, percutaneous ablation, particularly radiofrequency ablation using multiprobe bipolar technique, might be used with less limitations. This high percentage of untreated but potentially eligible patients emphasizes the need to extend these new and less invasive techniques; these techniques should benefit the patients, as there were previous decompensations of cirrhosis in $2 / 3$ of the cases. 
Such findings strengthen the importance of HCC screening in patients with cirrhosis; as a whole, screening has been shown to increase early tumor detection, the implementation of curative therapy, and overall survival (23).

However, our study has several main limitations. First, the median follow-up is still short $(<3$ years). Second, this cohort enrolled selected patients mainly: i) survivor 2 years from liver biopsy; ii) without significant residual consumption of alcohol at enrollment (abstinent 67.6\% or with a mild consumption of alcohol 16.9\%); iii) with mixed cause of cirrhosis (alcoholic and non-alcoholic steato hepatitis) as $35 \%$ had overweight and $32.5 \%$ were obese, with a significant proportion of metabolic syndrome (21\%). Accordingly, our population could be poorly representative of the whole population of alcoholic cirrhosis. Moreover, the absence of screening $\operatorname{logs}$ in all the participating centers avoids the assessment of selection bias. However, althougth a register of non-included patients was not designed, we observed in the main investigative center (Bondy), that among 446 potential eligible patients, only 141 (31.6\%) were enrolled. The main reasons to rule out 305 patients were liver failure (ChildPugh score $>6 ; n=85,27.8 \%)$, absence of histological proof of cirrhosis $(n=67,22.4 \%)$, presence of nodule suspect of HCC at baseline ( $n=36,12 \%)$, non-compliance to baseline visit $(n=31,10.4 \%)$, extra-hepatic life-threatening condition $(n=23,8 \%)$, positive serology for $\operatorname{HBV}$ or $\operatorname{HCV}(\mathrm{n}=21,7 \%)$, misunderstanding of French language $(\mathrm{n}=13,4.3 \%)$; refusal $(\mathrm{n}=$ $12,4 \%)$. In other centers, investigators claimed that their small number of included patients was related mainly to the absence of liver biopsy in the era of non-invasive tests for fibrosis diagnosis and the lack of human resources (dedicated clinical research staff), so that not all consecutive eligible patients may have been screened, possibly responsible for selection bias in estimates. Third, the included patients had to comply with a periodical follow-up, and they are among the most compliant of those referred for alcoholic cirrhosis. This compliance is explained by the large proportion of patients without significant ongoing alcohol consumption at enrollment. Thus, the periodic US screenings were accepted by most of the patients, with 
only $23 \%$ of patients discontinued at the study follow-up during the last 18 months. Sensitivity analyses assessed the impact of such dropouts, treating them as they have had an event (either death of HCC) occurred at the time of early withdrawal (worst scenario) or as they were free of any event at the time of scheduled follow-up (best scenario); the resulted range of 2-year survival estimates was from $82 \%$ (worst) to $93.7 \%$ (best), and that of 1 -year HCC incidence was from $1.4 \%$ (best) up to $7.3 \%$ (worst); however, the worst case was an unlikely scenario given at the time of last follow-up, there were actually alive and free of HCC. Moreover, of the 61 patients lost to follow-up on January, 2017 in the coordinating center (Bondy), 4 patients had developed a HCC and only 2 patients (3.2\%) had died including 1 with HCC; 41 (67\%) were still alive on March, 2018 including 3 who developed an HCC between January, 2017 and March, 2018. Otherwise, due to the small number of HCC, the analysis of risk factors for HCC development is weak. However, among identified risk factors, some are clinically interesting such as gender $(\mathrm{HR}=2.66)$ and age $(\mathrm{HR}=1.12)$, suggesting the possibility of restricting $\mathrm{HCC}$ surveillance to selected patients bearing a very high risk. Last, we only used multivariable survival models for defining prognostic factors based on univariable models, while other methods for variable selection could have been used; nevertheless, we were more interested in defining potential predictors of poor outcome rather than developing any prognostic score to be definetly computed; nevertheless, it should be validated in independent and large datasets before to be used in clinical settings. We used stepwise selection to avoid overfitting, and discarded all binary predictors with low prevalence as recommended. Our final model only retained 5 predictors, and given the 43 events, this appears reasonable with 8.6 events per variables in the range of 5-9 recommended by Vittinghoff et al. (24), so that the risk of overfitting appears low. Anyway, the risk of underfitting could not be discarded, owing to the low number of events that avoids the inclusion of additional terms in our model. 
In conclusion, in the CIRRAL French and Belgian prospective cohort, the HCC incidence is high (2.9 per 100 patients-year) suggesting that surveillance might be cost effective in patients with compensated Child-Pugh A alcohol-related cirrhosis. While a high proportion of the detected HCC cases were within the Milan criteria (77\%), only $56 \%$ of them were referred for curative treatments, emphasizing the need to increase access to percutaneous ablation for these fragile patients, who are usually not eligible for surgery.

\section{Acknowledgments}

This cohort was financed by the National Cancer Institute (INCa), the French Association for Research in Cancer and the National Agency for Research on HIV and Hepatitis.

Thanks to the 22 active investigative centers in France and Belgium and to the patients and their families.

This article is dedicated to the memory of Jean-Claude Trinchet for his help in the conception of this cohort and his outstanding contributions in liver cancer research during his career.

\section{References}

1. Ferlay J, Steliarova-Foucher E, Lortet-Tieulent J, Rosso S, Coebergh JW, Comber H, et al. Cancer incidence and mortality patterns in Europe: estimates for 40 countries in 2012. Eur J Cancer 2013;49:1374-403.

2. Rosa I, Denis J, Renard P, Lesgourgues B, Dobrin AS, Becker C, et al. A French multicentric longitudinal descriptive study of hepatocellular carcinoma management (the CHANGH cohort): preliminary results. J Hepatol 2010;52:S231-S2 (Abstract).

3. EASL-EORTC clinical practice guidelines: management of hepatocellular carcinoma. J Hepatol 2012;56:908-43.

4. Bruix J, Sherman M. Management of hepatocellular carcinoma. Hepatology 2005;42:1208-36.

5. Bruix J, Sherman M. Management of hepatocellular carcinoma: an update. Hepatology 2011;53:1020-2. 
6. EASL clinical practical guidelines : management of alcoholic liver disease. J Hepatol 2012;57:399-420.

7. Kansagara D, Papak J, Pasha AS, O'Neil M, Freeman M, Relevo R, et al. Screening for hepatocellular carcinoma in chronic liver disease: a systematic review. Ann Intern Med 2014;161:261-9.

8. Jepsen P, Ott P, Andersen PK, Sorensen HT, Vilstrup H. Risk for hepatocellular carcinoma in patients with alcoholic cirrhosis: a Danish nationwide cohort study.Ann Intern Med. 2012 Jun 19;156(12):841-7

9. West J, Card TR, Aithal GP, Fleming KM. Risk of hepatocellular carcinoma among individuals with different aetiologies of cirrhosis: a population-based cohort study. Aliment Pharmacol Ther 2017 Apr;45(7):983-990.

10. Mancebo A, Gonzalez-Dieguez ML, Cadahia V, Varela M, Perez R, Navascues CA, et al. Annual incidence of hepatocellular carcinoma among patients with alcoholic cirrhosis and identification of risk groups. Clin Gastroenterol Hepatol 2013;11:95-101.

11. Alberti KG, Zimmet P, Shaw J. The metabolic syndrome--a new worldwide definition. Lancet 2005;366:1059-62.

12. Liu Y. Sensitivity analyses for informative censoring in survival data: A trial example. J Biopharm Stat. 2017;27(4):595-610

13. Fujiwara N, Friedman SL, Goossens N, Hoshida Y. Risk factors and prevention of hepatocellular carcinoma in the era of precision medicine. J Hepatol. 2017 Oct 5. pii: S0168-8278(17)32328-0. doi: 10.1016/j.jhep.2017.09.016. [Epub ahead of print]

14. Hsu JY, Roy JA, Xie D, Yang W, Shou H, Anderson AH, Landis JR, et al. Statistical Methods for Cohort Studies of CKD: Survival Analysis in the Setting of Competing Risks. Clin J Am Soc Nephrol. 2017 Jul 7;12(7):1181-9. 
15. White IR, Royston P, Wood AM. Multiple imputation using chained equations: Issues and guidance for practice. Stat Med 2011;30:377-99.

16. Collins GS, Reitsma JB, Altman DG, Moons KGM. Transparent reporting of a multivariable prediction model for individual prognosis or diagnosis (TRIPOD): the TRIPOD statement. BMJ 2015; 162:55-64.

17. Trinchet JC, Bourcier V, Chaffaut C, Ait Ahmed M, Allam S, Marcellin P, et al. Complications and competing risks of death in compensated viral cirrhosis (ANRS CO12 CirVir prospective cohort). Hepatology 2015; 62:737-50

18. Nahon P, Bourcier V, Layese R, Audureau E, Cagnot C, Marcellin P, et al.Eradication of Hepatitis C Virus Infection in Patients With Cirrhosis Reduces Risk of Liver and Non-Liver Complications. Gastroenterology 2017;152:142-156.

19. van der Meer AJ, Feld JJ, Hofer H, Almasio PL, Calvaruso V, Fernandez-Rodriguez $\mathrm{CM}$, et al. Risk of cirrhosis-related complications in patients with advanced fibrosis following hepatitis C virus eradication. J Hepatol 2017;66:485-93.

20. Trinchet JC, Chaffaut C, Bourcier V, Degos F, Henrion J, Fontaine H, et al. Ultrasonographic surveillance of hepatocellular carcinoma in cirrhosis: a randomized trial comparing 3- and 6-month periodicities. Hepatology 2011;54:1987-97.

21. Costentin C, Goutte N, Sogni P. Patients with Alcohol Compared to Hcv-Related Hepatocellular Carcinoma (HCC) Have a Reduced Survival, Results of a Nationwide Study. Hepatology 2015;62 399 (Abstract).

22. Jepsen P, Lash TL, Vilstrup H. The clinical course of alcoholic cirrhosis: development of comorbid diseases. A Danish nationwide cohort study. Liver Int 2016;36:16961703.

23. Singal AG, Pillai A, Tiro J. Early detection, curative treatment, and survival rates for hepatocellular carcinoma surveillance in patients with cirrhosis: a meta-analysis. PLoS Med 2014;11:e1001624. 
24. Vittinghoff E, McCulloch CE. Relaxing the rule of ten events per variable in logistic and Cox regression. American Journal of Epidemiology, 2007;165(6):710-718. 
Figure 1. (1a) Geographic distribution of the 28 participating centers in the French territory and Belgium; (1b) Cumulative number of cohort inclusions between 2010 and 2015.

Figure 2: Flow chart for patient selection and history of focal liver lesion(s)

Figure 3: Cumulative incidence of focal liver lesions and hepatocellular carcinoma (HCC) in the whole cohort and in the subset of patients free of any focal lesion at study inclusion (3a), cumulative incidences of HCC and death free of HCC after the occurrence of the first focal liver lesion $(3 b)$

Figure 4: Patient outcomes in the CIRRAL cohort: Competing risks analysis of secondary outcomes, with cumulative incidence of decompensation, HCC before decompensation and death before decompensation and $\mathrm{HCC}$ (4a), overall survival (4b), survival after HCC diagnosis (4c) and cumulative incidence of deaths related and unrelated to liver disease (4d) 

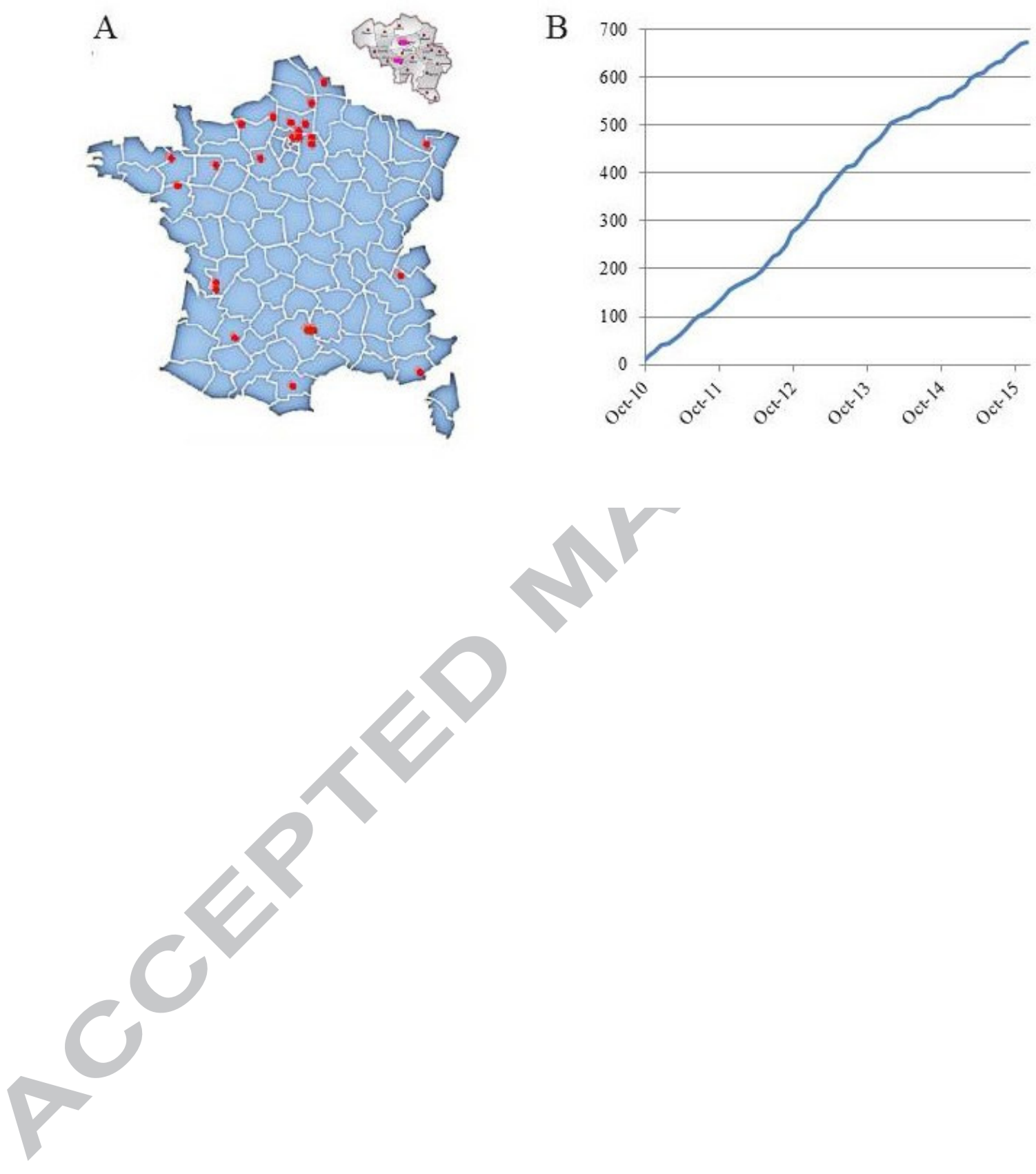


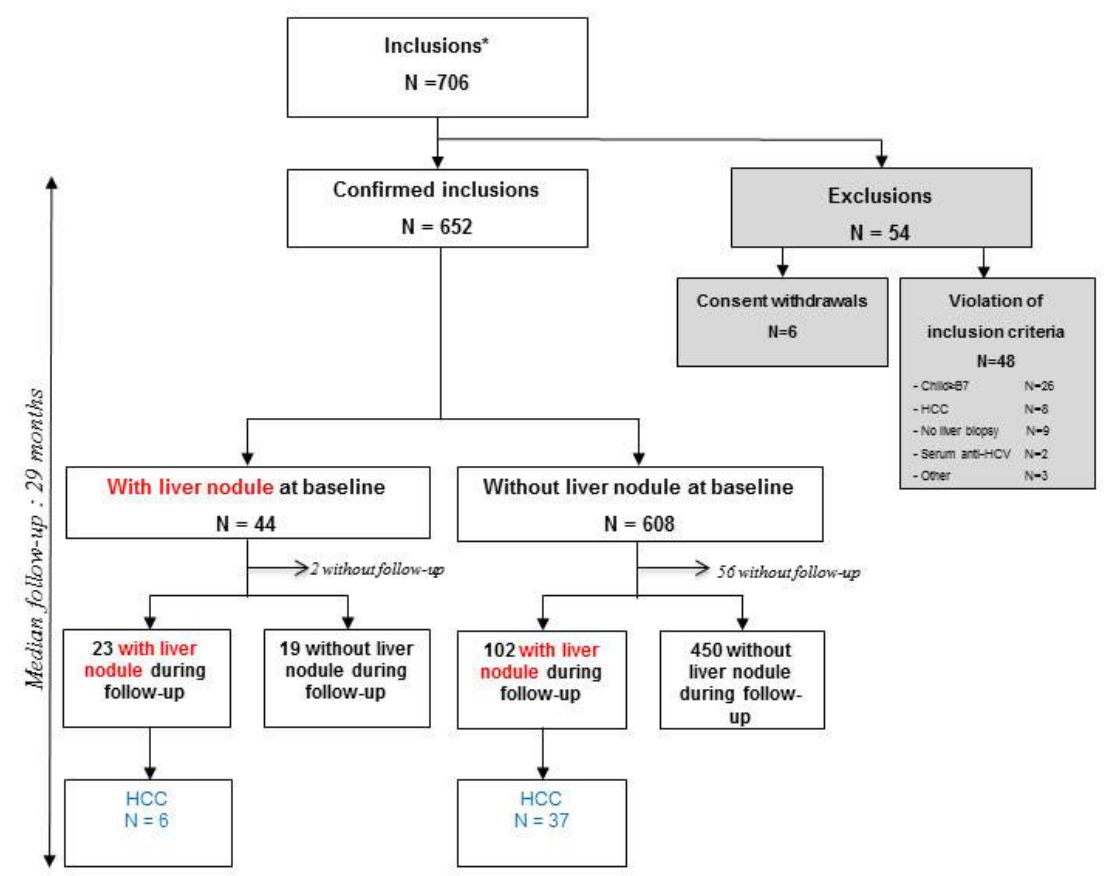

* Number of screened patients and of non included patients unknown 
$3 a$

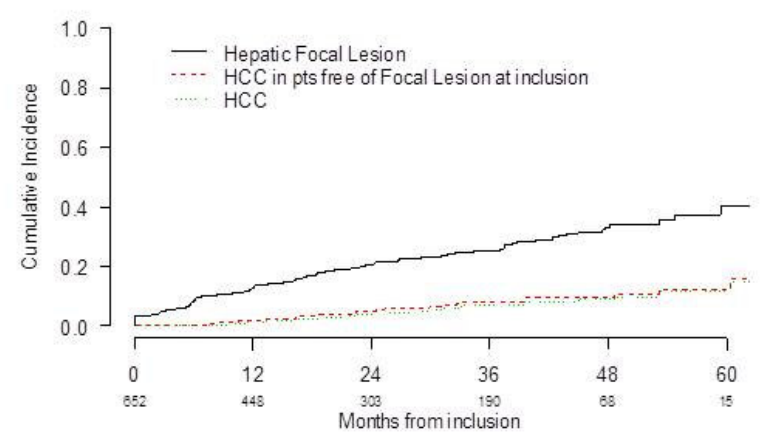

$3 b$
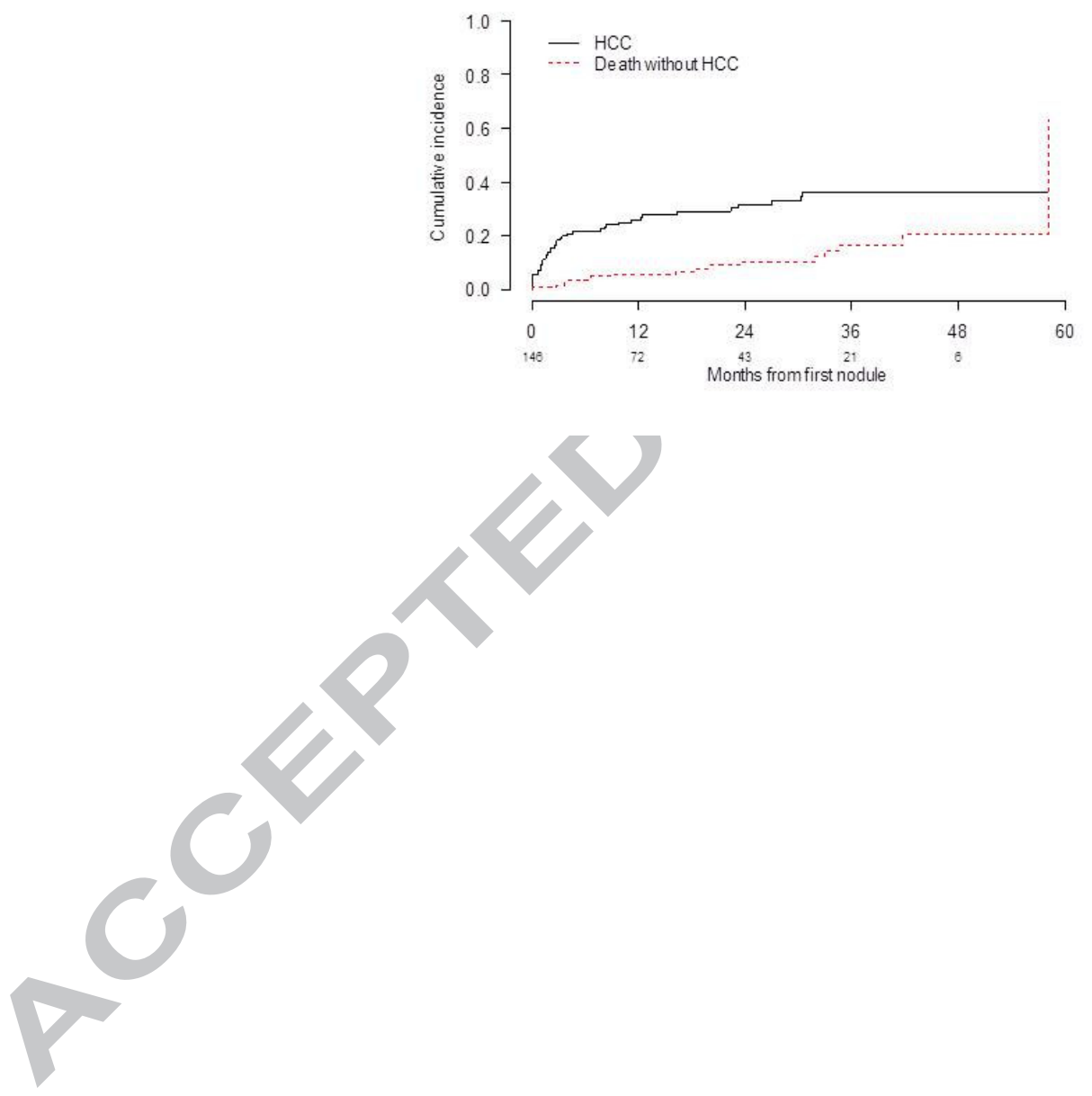
4a

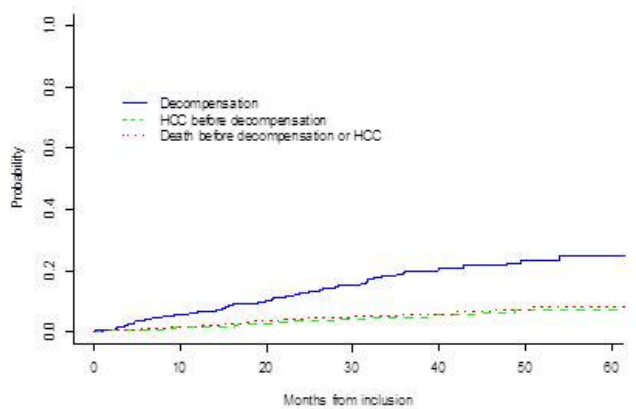

$4 c$

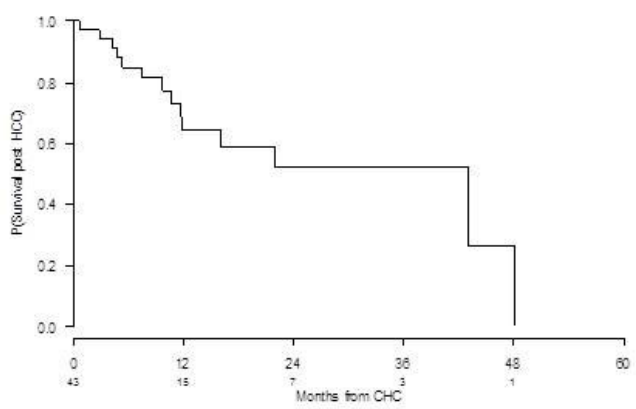

$4 b$

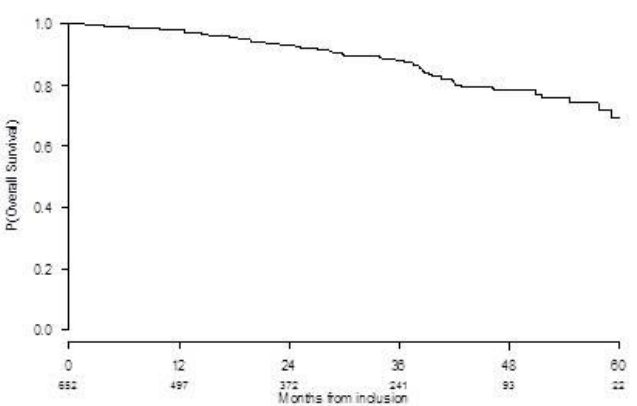

$4 d$

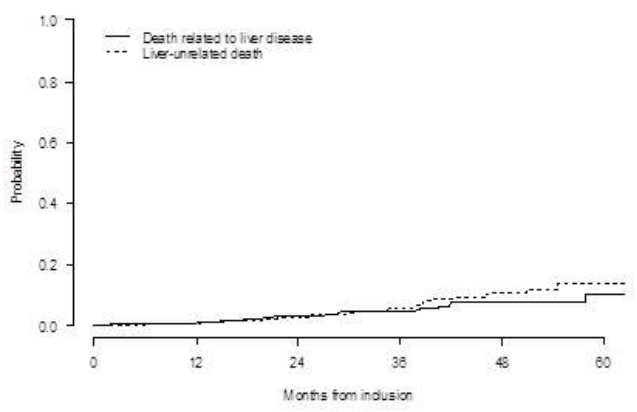

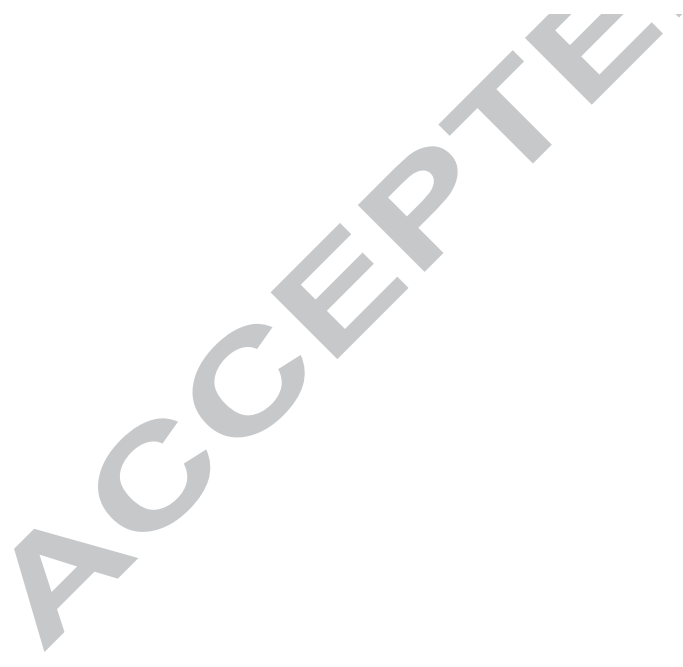


Table 1. Baseline characteristics of patients enrolled in the French and Belgian CIRRAL cohort

\begin{tabular}{cc} 
Characteristics & $\begin{array}{c}\text { Number of patients } \\
\text { with known } \\
\text { information }\end{array}$ \\
\hline
\end{tabular}

Age (years)

Male gender

History of cirrhosis

Duration of cirrhosis (months)

Previous decompensation

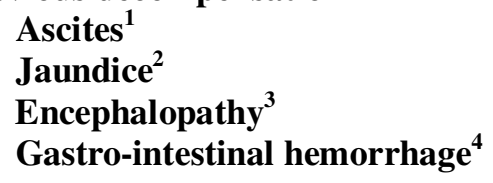

Tobacco consumption

Past

Active

Never

Coffee consumption

No

$<3$ cups / day

$\geq 3$ cups / day

Body mass index $\left(\mathrm{kg} / \mathrm{m}^{2}\right)$

Overweight / Obesity

$25-30 \mathrm{~kg} / \mathrm{m}^{2}$

$\geq 30 \mathrm{~kg} / \mathrm{m}^{2}$

Diabetes

Arterial hypertension
652

652

652

637

627

505

628

419

632

$$
\begin{gathered}
221(35 \%) \\
258(40.8 \%) \\
153(24.2 \%)
\end{gathered}
$$

555

619

$$
\begin{aligned}
& 128(23 \%) \\
& 267(48 \%) \\
& 160(29 \%)
\end{aligned}
$$

$27.5[24.2 ; 31.1]$

$220(35.5 \%)$

$201(32.5 \%)$

649

$146(22.5 \%)$

650

$271(41.7 \%)$ 
Hypertriglyceridemia

Metabolic syndrome

Prothrombin time (\%)

Bilirubin ( $\mu \mathrm{mol} / \mathrm{L})$

Serum albumin $(g / L)$

Platelet count $\left(10^{3} / \mathrm{mm}^{3}\right)$

AST (IU/L)

ALT (IU/L)

GGT (IU/L)

$\operatorname{AFP}(\mathbf{n g} / \mathrm{mL})$

Esophageal varices

None

Small

Medium

Voluminous

HIV co-infection

Positive

Negative

Not evaluated

Liver stiffness (fibroscan)

Evaluated

Not evaluated

Median value (KPa)

MELD Score
639

$63(9.9 \%)$

542

$114(21 \%)$

635

$78[67 ; 90]$

642

$14[10 ; 20]$

612

$40.6[37.1 ; 43.1]$

638

$145[104 ; 191.8]$

630

33 [26; 49]

633

$26[20 ; 39]$

635

109 [52; 217]

541

$3.8[2.5 ; 5.5]$

613

$304(49.6 \%)$

$190(31 \%)$

$98(16 \%)$

$21(3.4 \%)$

515

$1(0.2 \%)$

$371(72 \%)$

$143(27.8 \%)$

642

$269(41.9 \%)$

$373(58.6 \%)$

257

$24.5[14.1 ; 42.2]$

436

$8.5[7.5 ; 10.3]$

1. Ascites was defined by clinical symptoms with increased abdominal volume and paracentesis requirement; 2 . Jaundice was defined by increased bilirubin serum level over $100 \mu \mathrm{mol} / \mathrm{L} ; 3$. Encephalopathy was defined according international criteria (Hepatic Encephalopathy in Chronic Liver Disease: 2014 Practice Guideline by the European Association for the Study of the Liver and the American Association for the Study of Liver Diseases, J Hepatol 2014); 4. Gastro-intestinal bleeding was defined by overt hemorrhage in relation with Portal hypertension; 
Table 2. Characteristics of the detected hepatocellular carcinomas

\begin{tabular}{|c|c|c|}
\hline Characteristics & $\begin{array}{l}\text { Number of } \\
\text { patients } \\
\text { with known } \\
\text { information } \\
\end{array}$ & $\begin{array}{c}\text { N }(\%) \\
\text { Median [IQR] }\end{array}$ \\
\hline Number of HCC patients & 652 & $43(6.6 \%)$ \\
\hline $\begin{array}{l}\text { Tumor type } \\
\text { Uninodular } \\
2 \text { or } 3 \text { nodules } \\
>3 \text { nodules }\end{array}$ & 43 & $\begin{array}{l}25(58 \%) \\
15(35 \%) \\
3(7 \%)\end{array}$ \\
\hline $\begin{array}{l}\text { Diameter of largest nodule }(\mathbf{m m}) \\
\quad \leq 30 \\
31-50 \\
>50\end{array}$ & 43 & $\begin{array}{c}23[16 ; 29] \\
33(76 \%) \\
5(12 \%) \\
5(12 \%)\end{array}$ \\
\hline Portal thrombosis & 41 & $4(10 \%)$ \\
\hline $\begin{array}{l}\text { Milan Criteria } \\
\text { Fulfilled criteria } \\
\qquad 1 \text { nodule } \leq 50 \mathrm{~mm} \\
\quad 2 \text { or } 3 \text { nodules } \leq 30 \mathrm{~mm} \\
\text { Unfulfilled criteria }\end{array}$ & 43 & $\begin{array}{l}33(77 \%) \\
22(51 \%) \\
11(26 \%) \\
10(23 \%)\end{array}$ \\
\hline $\begin{array}{l}\text { AFP level at HCC diagnosis } \\
\text { (ng/mL) } \\
\text { Median [IQR] } \\
>200\end{array}$ & 42 & $\begin{array}{c}4.7[3.05 ; 14.6] \\
2(5 \%)\end{array}$ \\
\hline $\begin{array}{l}\text { HCC } \text { treatment }^{\dagger} \\
\text { Curative } \\
\text { Palliative or supportive care }\end{array}$ & 43 & $\begin{array}{l}24(56 \%) \\
19(44 \%)\end{array}$ \\
\hline $\begin{array}{c}\text { Survival status } \\
\text { Dead } \\
\text { Alive }\end{array}$ & 43 & $\begin{array}{l}14(33 \%) \\
29(67 \%)\end{array}$ \\
\hline
\end{tabular}

${ }^{\dagger} \mathrm{HCC}$ management may include one or several of the associated therapeutic procedures. HCC: hepatocellular carcinoma. IQR: interquartile rang 
Table 3. Prognostic factors for hepatocellular carcinoma (HCC)

\begin{tabular}{|c|c|c|c|c|c|c|}
\hline & \multirow{2}{*}{$\begin{array}{l}\text { Number of } \\
\text { patients } \\
\text { with known } \\
\text { information }\end{array}$} & \multicolumn{3}{|c|}{ Univariate analyses } & \multicolumn{2}{|c|}{ Multivariate analysis } \\
\hline & & $\begin{array}{c}\text { No HCC } \\
N=609\end{array}$ & $\begin{array}{l}\mathrm{HCC} \\
\mathrm{N}=43\end{array}$ & P-value* & HR (95\% CI) & P-value \\
\hline Age (years) & 652 & $57.9[50.6 ; 63.7]$ & $66[61 ; 72.4]$ & $<0.0001$ & $1.12(1.07 ; 1.18)$ & $<0.0001$ \\
\hline $\begin{array}{l}\text { Body Mass Index } \\
\left(\mathrm{kg} / \mathrm{m}^{2}\right)\end{array}$ & 619 & $27.4[24.2 ; 30.8]$ & $30.1[27.1 ; 33.3]$ & 0.007 & & \\
\hline Male gender & 652 & $404(66.3 \%)$ & $36(83.7 \%)$ & 0.02 & $2.66(1.12$ & 0.027 \\
\hline Diabetes & 649 & $130(21.4 \%)$ & $16(38 \%)$ & 0.035 & & \\
\hline Arterial hypertension & 650 & $244(40.2 \%)$ & $27(62.8 \%)$ & 0.027 & & \\
\hline Alcohol withdrawal & 652 & $421(69.1 \%)$ & $20(46.5 \%)$ & 0.004 & & \\
\hline Coffee $\geq 3$ cups/ day & 555 & $154(25.3 \%)$ & $6(14 \%)$ & 0.035 & & \\
\hline $\begin{array}{l}\text { Previous } \\
\text { decompensation }\end{array}$ & 637 & $390(64.0 \%)$ & $26(60.5 \%)$ & 0.67 & & \\
\hline $\mathbf{A F P}(\mathrm{ng} / \mathrm{mL})$ & 541 & $3.7[2.5 ; 5.2]$ & $5.2[3.3 ; 6.9]$ & $<0.0001$ & $1.07(1.02 ; 1.12)$ & 0.004 \\
\hline $\begin{array}{l}\text { Prothrombin time } \\
(\%)\end{array}$ & 635 & $79[68 ; 91]$ & $76[63 ; 78]$ & 0.001 & $0.97(0.94 ; 0.996)$ & 0.025 \\
\hline Bilirubin $(\mu \mathrm{mol} / \mathrm{L})$ & 642 & $13.7[9.2 ; 19.3]$ & $20[15 ; 27.4]$ & $<0.0001$ & $1.06(1.02 ; 1.096)$ & 0.005 \\
\hline Serum albumin $(g / L)$ & 612 & $40.8[37.4 ; 43.6]$ & $38[35.5 ; 41]$ & $<0.0001$ & & \\
\hline $\begin{array}{l}\text { Platelet count } \\
\left(10^{3} / \mathrm{mm} 3\right)\end{array}$ & 638 & 05;194] & $108[76 ; 151]$ & 0.0004 & & \\
\hline
\end{tabular}

* by the two-sided log-rank 


\section{Graphical abstract}

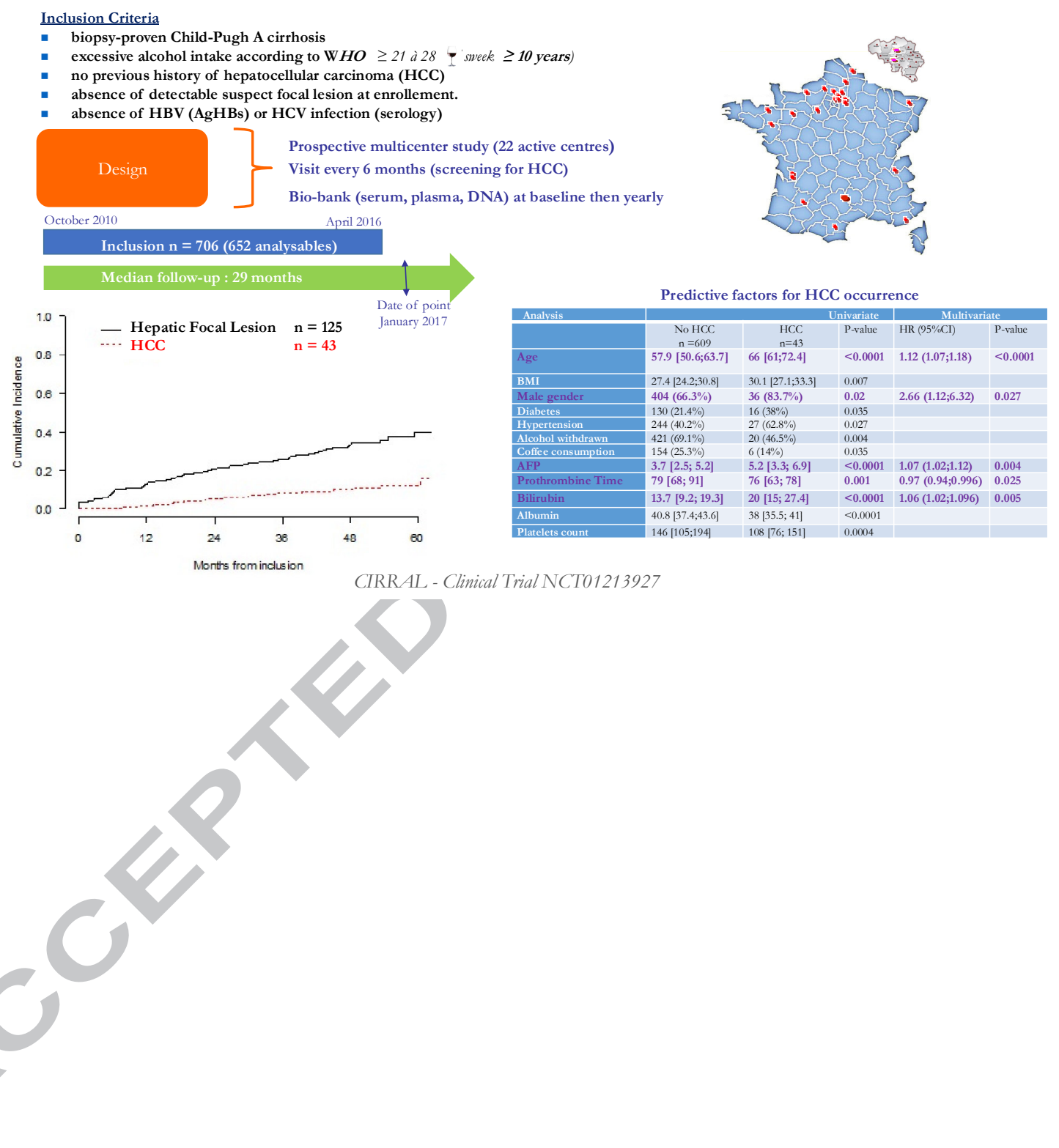




\section{Highlights}

- $\mathrm{HCC}$ annual incidence is $2.9 \%$, emphazising the need for its screening in alcoholic cirrhosis.

- The independent risk factors for HCC is age, male gender, baseline AFP and biological surrogate markers of minimal liver failure

- Only $56 \%$ of the patients diagnosed with HCC underwent a curative treatment

- The main reasons not to treat were liver failure and/or impaired general status at diagnosis.

- Our results highlight the need to spread the practice of radiological ablative therapies for patients with alcoholic cirrhosis, who remain the vast majority of patients who will develop HCC in Europe. 